\title{
Double Primary Epidermoid Carcinoma of the Vulva and Cervix
}

\author{
Yew Cheong Choo, M.D. and George W. Morley, M.D. \\ Division of Gynecologic Oncology, Department of Obstetrics and Gynecology, University of \\ Michigan Hospital, Ann Arbor, Michigan 48109
}

Received July 16, 1979

\begin{abstract}
A retrospective study was undertaken on patients with multiprimaries associated with vulva carcinoma, analyzing in particular 33 patients with double epidermoid primaries of the vulva and cervix. It is noted that $20.8 \%$ of patients with vulvar carcinoma had a second primary; this occurred more frequently in intraepithelial $(33.3 \%)$ than in invasive $(16.0 \%)$ vulvar carcinoma. While the commonest second primary is located in the cervix, this association appears significant only in patients with intraepithelial $(22.5 \%)$, but not in invasive (7.9\%), vulvar carcinoma. The incidences previously reported in the literature are also reviewed for comparison.
\end{abstract}

\section{INTRODUCTION}

That epidermoid carcinoma of the vulva often is associated with a second primary was first noted by Taussig [1] in 1940. This relationship has subsequently been reported by numerous authors, with varying frequencies, both for intraepithelial as well as invasive carcinoma. Abell [2], in 1960, suggested that a second primary occurred more frequently in intraepithelial than in invasive carcinoma of the vulva. More specifically, a separate primary in the cervix, either synchronous or metachronous with that in the vulva, appears especially significant. Also, an appreciable number of vulvar carcinomas have been reported following radiotherapy for cervical carcinoma [3-5]. Jimerson and Merrill [5] in 1970 collected 132 patients with multiprimary epidermoid carcinomas involving both the cervix and vulva, including 15 cases of their own. As yet, however, there has not been an in-depth study of multiprimaries associated with vulvar carcinoma. We consider it appropriate to focus attention on these issues and to present our experiences at the University of Michigan Hospital. Reports in the literature of this relationship are reviewed for the purpose of comparison.

\section{MATERIALS AND METHODS}

Data on all patients with multiprimaries of the female genital tract for a 20-year period between January 1957 and December 1977 were obtained from the University of Michigan Hospital Tumor Registry. Eighty-four patients were found to have multiprimaries associated with the vulva; these patients were studied. During this same period, 5386 patients with female genital tract cancers were treated at this Medical Center. This included 403 patients with epidermoid vulvar car- 
TABLE 1

Sites of Multiprimaries in Patients with Intraepithelial Carcinoma of the Vulva

\begin{tabular}{lc}
\hline Cervix & \\
Skin & 25 \\
Bladder & 3 \\
Colon & 2 \\
Lymphoma & 1 \\
Vagina & 1 \\
Trophoblastic disease & 1 \\
Breast & 1 \\
Unclassified & 1 \\
Total & 2 \\
\hline
\end{tabular}

${ }^{a}$ Ten patients had additional primary or primaries in the vagina, perineum, or anus.

cinoma, 111 of whom had intraepithelial, and 292, invasive carcinoma. Cervical carcinoma totaled 2800 in number.

\section{RESULTS}

The locations of various primaries in these 84 patients with intraepithelial and invasive carcinoma of the vulva are listed in Tables 1 and 2 . Thus, 37 patients with intraepithelial, and 47 patients with invasive carcinoma of the vulva had another primary or primaries, representing an incidence of 33.3 and $16.0 \%$, respectively. Cervical carcinoma was the most common second primary, however. This occurred in 25 patients with intraepithelial, and 23 patients with invasive carcinoma of the vulva comprising, respectively, 67.5 and $48.7 \%$ of multiprimaries in each group. Fifteen patients with double primaries in the cervix and vulva had additional primary or primaries in the vagina, perineum, and anus. The remaining 33 patients are presented below.

TABLE 2

Sites of Multiprimaries in Patients with Invasive Carcinoma of the Vulva

\begin{tabular}{lr}
\hline & 23 \\
Cervix & \\
Skin & 5 \\
Ovary & 2 \\
Bladder & 2 \\
Uterus & 2 \\
Lung & 2 \\
Breast & 2 \\
Small intestine & 1 \\
Appendix & 1 \\
Hodgkin & 1 \\
Pancreas & 1 \\
Urethra & 1 \\
Rectum & 1 \\
Unclassified & 3 \\
Total & 47 \\
\hline
\end{tabular}

${ }^{a}$ Five patients had additional primary or primaries in the vulva, perineum, or anus. 


\section{Synchronous Primaries of the Cervix and Vulva}

Ten patients had simultaneous primaries in the cervix and vulva (Table 3). Only 1 patient had an intraepithelial lesion in both locations, while the rest had intraepithelial-invasive combinations. Their age disparity is also evident (Table 3).

The presenting symptom varied. Of 6 patients with intraepithelial carcinoma of the vulva and invasive carcinoma of the cervix, 2 presented with pruritus vulva, 2 with vaginal bleeding, 1 with vaginal discharge, and the sixth was asymptomatic. Of 3 patients with invasive carcinoma of the vulva and in situ carcinoma of the cervix, 2 complained of a vulvar swelling, and 1, vaginal bleeding. The patient with simultaneous intraepithelial disease of the cervix and the vulva was asymptomatic.

All patients with carcinoma of the vulva, whether intraepithelial or invasive, had visible lesions. These were variously described as: leukoplakia, ulcer, mass, red, or granular lesions. On the contrary, three of four patients with in situ carcinoma of the cervix had no abnormal findings; the diagnosis in all was made following an abnormal cervical smear. Among six patients with invasive carcinoma of the cervix, biopsy of a suspicious lesion in five yielded a diagnosis. The sixth patient underwent a diagnostic conization for an abnormal cervical cytology, this showed carcinoma in situ; however, following a total abdominal hysterectomy and bilateral salpingo-oophorectomy, a focus of invasive cervical squamous carcinoma was found.

With the exception of a stage IIIB cervical carcinoma, the invasive primary of the vulva or the cervix in these patients was in stage I.

Of 6 patients with invasive cervical carcinoma and intraepithelial vulvar disease, 4 received radiotherapy for the cervical lesion and surgery, ranging from wide local excision to radical vulvectomy, for the vulvar disease. One patient with stage IIIB cervical carcinoma died of metastases to the brain while undergoing radiation. The sixth patient underwent trachelectomy for a stage IA cervical carcinoma and simultaneous radical vulvectomy for intraepithelial vulvar disease; she however died of postoperative sepsis and acute renal failure. Of 3 patients with invasive vulvar and intraepithelial cervical carcinoma, 1 was treated with only a hemivulvectomy because of her advanced age, while her cervical lesion was followed. She died 9 years later from a myocardial infarction. Two patients were treated sequentially with a radical vulvectomy and groin lymphadenectomy followed by a total abdominal hysterectomy and bilateral salpingo-oophorectomy. The patient with double intraepithelial carcinoma of the cervix and vulva under-

TABLE 3

Synchronous Double Primaries of the Cervix and the Vulva

\begin{tabular}{lccc}
\hline Vulvar carcinoma & Cervical carcinoma & $\begin{array}{c}\text { No. of } \\
\text { patients }\end{array}$ & $\begin{array}{c}\text { Median age } \\
\text { (years) }\end{array}$ \\
\hline In situ & Invasive & 6 & 58 \\
Invasive & In situ & 3 & 57 \\
In situ & In situ & 1 & 24 \\
\hline
\end{tabular}


went therapeutic conization as well as a wide excision of her vulvar lesion. In all, 7 patients are alive without disease 1 to 29 years after treatment.

\section{Metachronous Primaries of Cervix and Vulva}

There are 23 patients in this group as shown in Table 4 . Significantly the initial primary is cervical in all but 1 patient. Of the 12 patients with an initial invasive cervical primary, 6 developed an intraepithelial and 6 , invasive vulvar carcinoma after a mean interval of 10 and 17.3 years respectively. It is noteworthy that 10 among them had prior radiation treatment for cervical cancer.

The stages of the initial invasive cervical carcinoma in these 12 patients included 7 stage I and 1 stage II disease. The remaining 4 received treatment elsewhere and had no detailed information available. Of the 6 patients who subsequently developed an invasive primary in the vulva, 5 had stage I and 1 had stage IV disease.

Four of the six patients with sequential invasive, cervical, and vulvar carcinoma underwent radical vulvectomy and bilateral groin lymphadenectomy for their vulvar disease. Two of them died-one from postoperative renal failure and the other from recurrent metastatic vulvar disease. One had local recurrence, a planned exenteration was abandoned on finding positive iliac nodes; she subsequently received further radiation to the pelvis, groins and perineum and is well 2 years later. The fifth patient refused either surgery or radiotherapy, and is alive with disease 1 year after diagnosis. The sixth patient died of an advanced stage IV vulvar disease 1 year after chemotherapy.

In six patients with subsequent intraepithelial vulvar carcinoma following an invasive cervical carcinoma, surgery ranging from conservative wide local excision to radical vulvectomy was performed. Five are without disease from 6-9 years. One went on to develop a third primary in the esophagus 3 years later and succumbed to it.

Nine patients had sequential intraepithelial carcinoma involving the cervix and then the vulva. These patients were relatively young with a mean age of 30.2 at initial diagnosis and progressed to develop a second primary at a relatively short interval averaging 4 years. Conservative treatment was the rule in these patients. Wide local excision of vulvar lesion was performed in 6 patients, subtotal vulvectomy in 2 , and local $5 \mathrm{FU}$ therapy in 1 . All are without disease from 2 to 25 years.

One patient developed an invasive vulvar carcinoma 20 years after an initial

TABLE 4

Metachronous Double Primaries of the Cervix and the Vulva

\begin{tabular}{llcc}
\hline Initial primary & Second primary & $\begin{array}{c}\text { No. of } \\
\text { patients }\end{array}$ & $\begin{array}{c}\text { Mean interval } \\
\text { (years) }\end{array}$ \\
\hdashline- & & - \\
Invasive $\mathrm{Ca}$, cervix & Invasive Ca, vulva & 6 & 17.3 \\
Invasive Ca, cervix & In situ $\mathrm{Ca}$, vulva & 6 & 10.0 \\
In situ $\mathrm{Ca}$, cervix & In situ $\mathrm{Ca}$, vulva & 9 & 4.0 \\
In situ $\mathrm{Ca}$, cervix & Invasive $\mathrm{Ca}$, vulva & 1 & 20.0 \\
Invasive $\mathrm{Ca}$, vulva & In situ $\mathrm{Ca}$, cervix & 1 & 8.0 \\
\hline
\end{tabular}


intraepithelial carcinoma in the cervix. She died 5 years after a radical vulvectomy and groin lymphadenectomy from a myocardial infarction.

The only patient who had a preceding invasive vulvar carcinoma acquired an intraepithelial carcinoma of the cervix $\mathbf{8}$ years after radical vulvectomy and groin lymphadenectomy. A total abdominal hysterectomy and bilateral salpingooophorectomy were performed and she is alive 18 years later.

\section{DISCUSSION}

The incidence of multiple primary neoplasms in the general population has been reported to vary from less than $1 \%$ [6] to $11 \%$ [7], but averages 3-5\% in most clinical and autopsy series. Moertel showed that multicentric multiprimary neoplasms tend to occur more frequently in the same tissues and organs, and in bilaterally paired organs. Multiprimaries in nonidentical tissues or organs, on the other hand, showed no statistical increase in frequency. These occur, rather, by chance related to age, longevity, and cure of the initial primary $[8,9]$.

The entire lower genital tract, including the anus and the perineum, are embryologically interrelated. It is postulated that a common carcinogen may affect the unique epithelial field of this region in a manner resulting in simultaneous or sequential development of neoplasms $[4,10]$. A viral carcinogen may be operative, and implicated are HSV II [11,12], as well as condyloma accumulata viruses [13]. A venereal route may explain the high frequency of involvement of the cervix and vulva, both being the commonest neoplasms of the lower genital tract.

Since the previous reviews by Hansen and Collins in 1967 [14], and Jimerson and Merrill in 1970 [5], there have been continual reports of double primaries of the cervix and vulva. An update of these cases brings the total number to 285 (Table 5).

The reported incidence of a second primary in patients with vulvar carcinoma ranges widely from $1.5 \%$ [25] to $37.5 \%$ [2]. An overall incidence of $20.8 \%$ is found

TABLE 5

Reported Cases of Douri.f. Primarifs of the Cervix and the Vulva

\begin{tabular}{lcc}
\multicolumn{1}{c}{ Author } & No. of cases & Prior radiation \\
\hline Jimerson and Merrill [5], 1970 & 132 & 28 \\
Collins [15], 1970 & 8 & 3 \\
Hughes [16], 1971 & 7 & 1 \\
Boutselis [17], 1972 & 4 & \\
Boutselis [18], 1972 & 7 & \\
Charles [19], 1972 & 1 & \\
Kelly [20], 1972 & 2 & 3 \\
Franklin [11], 1972 & 33 & \\
Woodruff [13], 1973 & 8 & \\
Dean [21], 1974 & 9 & \\
Parker [22], 1975 & 6 & \\
Japaze [23], 1976 & 23 & 10 \\
Buchler [24], 1977 & 12 & 45 \\
Present series & 33 & \\
Total & 285 & \\
\hline
\end{tabular}


TABLE 6

Reported Incidence of Second Primary in Intraepithelial Carcinoma of the Vulva

$\begin{array}{ll}\text { Abell [2], 1961 } & 37.5 \% \\ \text { Boutselis [17], 1972 } & 29.1 \% \\ \text { Japaze [23], 1977 } & 40.8 \% \\ \text { Present series } & 33.3 \% \\ \text { Average } & 35.2 \%\end{array}$

in our series. This occurred more frequently in patients with intraepithelial (33.5\%) than with invasive carcinoma $(16.0 \%)$, and is in concurrence with most reported incidences (Tables 6 and 7). In our series, nongenital second primaries comprise an incidence of 9.0 and $6.9 \%$, respectively, in patients with intraepithelial and invasive carcinomas of the vulva. These values are not significantly higher than the reported incidence of multiprimary neoplasms in the general population. More important, however, is the occurrence of a cervical primary in patients with vulvar carcinoma. Our findings indicate that this association is significant in patients with intraepithelial $(22.5 \%)$ but not with invasive vulvar carcinoma (7.9\%). The reported incidence of cervical carcinoma in patients with intraepithelial and invasive carcinomas of the vulva are presented for comparison in Tables 8 and 9 . Where reports in both groups by the same author are available, our findings are in agreement with that of Boutselis $[17,18]$ and Japaze [23], but at variance with that of Merrill [31].

The significant association of cervical neoplasm with intraepithelial vulvar carcinoma should be borne in mind. Lately, intraepithelial carcinoma of the vulva has been reported to increase in frequency $[13,15,17,23,30,31]$, afflicting more young females than before [23]; some have noted an absolute increase in relationship to its invasive counterpart $[15,17,31,32]$ suggesting perhaps a changing trend in this disease. This apparent increase may also result from increased awareness by both physicians and patients, as well as freer use of biopsies diagnosing more cases in the preinvasive state.

In invasive double primaries of common epithelial origin as in the cervix and the vulva, differentiation from metastases is important. Warren and Gates laid down criteria for multiprimaries that are generally accepted: that each of the tumors be

TABLE 7

Reported Incidence of Second Primary in Invasive Carcinoma of the Vulva

\begin{tabular}{lr}
\hline Taussig [1], 1940 & $6.4 \%$ \\
Green [26], 1958 & $13.4 \%$ \\
Gosling [25], 1961 & $1.5 \%$ \\
Collins [27], 1963 & $15.6 \%$ \\
Shingleton [28], 1970 & $15.0 \%$ \\
Cavanagh [29], 1970 & $5.0 \%$ \\
Boutselis [18], 1972 & $15.0 \%$ \\
Parker [22], 1975 & $22.0 \%$ \\
Japaze [23], 1977 & $14.0 \%$ \\
Present series & $16.0 \%$ \\
Average & $13.3 \%$ \\
\hline
\end{tabular}


TABLE 8

Rfported Incidfnce of Cervical Neoplasm in Intraepithelial Carcinoma of the Vulva

$\begin{array}{ll}\text { Hansen [14], 1967 } & 25.0 \% \\ \text { Rutledge [30], 1968 } & 26.8 \% \\ \text { Boutselis [17], 1972 } & 16.7 \% \\ \text { Merrill [31], 1973 } & 55.5 \% \\ \text { Japaze [23], 1977 } & 28.2 \% \\ \text { Present series } & 22.5 \% \\ \text { Average } & 29.1 \%\end{array}$

present with definite pictures of malignancy, that each be distinct, and that the probability of one being a metastasis of the other be excluded [33]. In our series, metastatic carcinoma of the cervix to the vulva has been excluded, and simultaneous invasive carcinoma of the cervix and vulva is conspicuously absent. Although there were six patients with invasive cervical carcinoma who subsequently developed an invasive vulvar cancer, their mean interval was 17.3 years.

It is noteworthy that of the 12 patients who developed a vulvar primary subsequent to an invasive cervical cancer, 10 received prior radiation, with a mean interval of 17.5 years. There is undoubtedly a strong implication of radiation carcinogenicity in our series. Approximately one-third of our patients with double primaries of cervix and vulva had prior radiation for cervical carcinoma. This also has been the experience of Collins [15], Woodruff [13], and Figge [3]. Of 285 patients with double primaries of the cervix and vulva reported to date, 45 had prior radiotherapy for cervical cancer (Table 6). The vulvar skin is known to be low in radiotolerance; this perhaps is attributable to the warmth and moisture in this area, as well as the blood supply to the skin being derived from end arteries. Although the vulvar skin is excluded from the portals of radiation treatment for cervical carcinoma, it nevertheless receives approximately $5 \%$ of scattered external radiation, and a much higher dose is contributed by the sources in the tandem-ovoid system. The relationship between the given dose and the dose received by the vulva cannot be estimated from our scries. It has becn known that radiation-induced carcinoma of the skin may occur following chronic exposure [34], or from an area of radiodermatitis [35]. Thus, possibly the delicate vulvar skin is sensitive to radiation carcinogenicity, or radiation itself potentiates preexisting local carcinogens in the vulva.

Awareness of a possible double primary in the cervix and vulva is the keystone to the diagnosis of these related entities. Three of our nine patients with syn-

TABLE 9

Reported Incidence of Cervical Neoplasm in Invasive Carcinoma of the Vulva

Boutselis [18], 1972

Merrill [31], 1973

Dean [21], 1974

Japaze [23], 1977

Present series

Average
$7.8 \%$

$35.0 \%$

$20.9 \%$

$2.5 \%$

$7.9 \%$

$14.8 \%$ 
chronous primaries probably failed to have their intraepithelial vulvar lesions detected until radiation treatment was begun. Such patients may present with a symptom referable to either location, or they may be entirely asymptomatic. Premalignant lesions of the cervix often arouse suspicion initially from an abnormal cervical smear, and the majority of such patients will reveal no gross findings in the cervix. On the contrary, in vulvar carcinoma, whether intraepithelial or invasive, a lesion is invariably present. It thus cannot be overemphasized that a meticulous visual examination of the external genitalia, coupled with a liberal biopsy of any suspicious areas, is the only means of detecting its lesions. Toluidine blue staining of the vulva may not be reliable as areas of fissures, cracks, and abrasions retain dyes, and a false positive rate of $17 \%$ has been reported [36]. To avoid this error, a colposcope could be used before and after staining [37].

In the case of the cervix, Schiller staining may be equally inaccurate [38]. In experienced hands, the colposcope should be a highly reliable and accurate tool in evaluating and screening the entire lower genital tract for preinvasive and invasive diseases.

Our series also indicates that in patients with invasive vulvar carcinoma, extensive workup, other than that essential for staging purposes, on the pretext of diagnosing associated nongenital primaries is not warranted.

The presence of a vulvar primary should always arouse suspicion of a possible simultaneous primary in the cervix. Whereas in metachronous primaries of the cervix and vulva the initial primary is invariably cervical; thus in the followup of patients with cervical carcinoma, one should be on the lookout for a possible new primary in the vulva.

Not uncommonly, an additional primary may also occur in the vagina, perineum, or anus. This rightly is so, since the entire region is at risk for multicentric multiprimaries.

The treatment modality is as conventional as for the individual neoplasm alone. In preinvasive lesions, conservative treatment can and should be employed, especially in younger patients, provided an adequate clear margin of resection can be obtained. Mapping techniques using staining methods and/or colposcopy can be utilized. In the presence of simultaneous invasive cervical and vulvar carcinoma, if radiation is contemplated for the cervical lesion, treatment of the vulvar lesion should probably take precedence over that of cervical cancer so as to avoid morbid sequelae of compromised wound healing. Premalignant lesions are curable by conservative methods, and, if they recur, can be retreated. The presence of double primaries in the cervix and vulva does not appear to adversely affect prognosis or survival.

\section{REFERENCES}

1. Taussig, F. L. Cancer of vulva; analysis of 155 cases (1911-1940), Amer. J. Obstet. Gynecol. 40, 764-779 (1940).

2. Abell, M. R., and Gosling, J. R. G. Intraepithelial and infiltrative carcinoma of vulva: Bowenoid type, Cancer 14, 318-329 (1961).

3. Figge, D. C., and Gaudenz, R. Invasive carcinoma of vulva, Amer. J. Obstet. Gynecol. 119, 382-394 (1974). 
4. Marcus, S. L. Multiple squamous cell carcinoma involving the cervix, vagina, and vulva: The theory of multicentric origin, Amer. J. Obstet. Gynecol. 80, 802-811 (1960).

5. Jimerson, G. K., and Merrill, J. A. Multicentric squamous malignancy involving both cervix and vulva, Cancer 26, 150-153 (1970).

6. Haddow, A. J., and Boyd, J. F. Multiple primary malignant neoplasm in western hospital region, Scotland: A survey based on cancer registration data, Scot. Med. J. 17, 143-152 (1973).

7. Pickren, J. W. Cancer often strikes twice, N.Y. J. Med. 63, 95-99 (1965).

8. Moertel, C. G., Dokerty, M. B., and Baggenstoss, A. H. Multiple primary malignant neoplasms, Cancer 14, 221-248 (1961).

9. Moertel, C. G. Multiple primary malignant neoplasms. Historical aspects, Cancer 40, 1786-1792 (1977).

10. Newman, W., Cromer, J. K. The multicentric origin of carcinoma arising in female ano-genital tract, Surg. Gynecol. Obstet. 108, 273-281 (1970).

11. Franklin, E. R., and Rutledge, F. D. Epidemiology of epidermoid carcinoma of valus, Obstet. Gynecol. 39, 165-172 (1972).

12. Josey, W. G., Nahmias, A. J., and Naib, Z. M. Virus and cancer of the lower genital tract, Cancer 38, 526-533 (1976).

13. Woodruff, J. D., Julian, C., Puray, T., Mermut, S., and Katayama, P. The contemporary challenge of carcinoma in in situ of vulva, Amer. J. Obstet. Gynecol. 115, 677-686 (1973).

14. Hansen, L. H., and Collins, C. G. Multicentric squamous cell carcinoma of lower genital tract. 11 cases with both epidermoid carcinoma of both vulva and cervix, Amer. J. Obstet. Gynecol. 98, 982-986 (1967).

15. Collins, C. G., Roman-Lopez, J. J., and Lee, F. Y. L. Intraepithelial carcinoma of vulva, Amer. J. Obstet. Gynecol. 108, 1187-1191 (1970).

16. Hughes, R. R. Early diagnosis and management of premalignant lesion and early invasive cancer of vulva, S. Med. J. 64, 1490-1492 (1971).

17. Bout selis, J. G. Intraepithelial carcinoma of vulva, Amer. J. Ohstet. Gynerol. 113, 733-738 (1972).

18. Boutselis, J. G. Radical vulvectomy for invasive squamous cell carcinoma of vulva, Obstet. Gynecol. 39, 827-836 (1972).

19. Charles, A. H. Carcinoma of vulva, Brit. Med. J. 1, 397-402 (1972).

20. Kelly, J. Malignant disease of vulva, J. Obstet. Gynecol. Brit. Commonw. 79, 265-272 (1972).

21. Dean, R. E., Taylor, E. S., Weisbrod, D. M., and Martin, J. W. The treatment of premalignant and malignant lesions of the vulva, Amer. J. Obstet. Gynecol. 119, 59-68 (1974).

22. Parker, R. T., Duncun, I., Rampone, J., and Creasman, W. Operative management of early invasive epidermoid carcinoma of vulva, Amer. J. Obstet. Gynecol. 123, 349-355 (1975).

23. Japaze, H., Garcia-Bunnell, R., and Woodruff, J. D. Primary vulvar cancer. A review of in situ and invasive carcinoma, 1935-1972, Obstet. Gynecol. 49, 404-411 (1976).

24. Buchler, D. A. Multiple primaries and gynecological malignancies, Amer. J. Obstet. Gynecol. 123, 376-381 (1977).

25. Gosling, J. R. G., Abell, M. R., Drolette, B. M., and Loughrim, T. D. Infiltrative squamous cell (epidermoid) carcinoma of vulva, Cancer 14, 330-343 (1961).

26. Green, T. H., Ulfelder, H., and Meigs, J. V. Epidermoid carcinoma of vulva: An analysis of 238 cases. 1. Etiology and diagnosis, Amer. J. Obstet. Gynecol. 75, 834-847 (1958).

27. Collins, C. G., Collins, J. H., Barclay, D. L., and Nelson, E. W. Cancer involving the vulva. A report of 109 consecutive cases, Amer. J. Obstet. Gynecol. 87, 762-772 (1963).

28. Shingleton, H. M., Fowler, W. L., Palumbo, L., and Koch, C. G. Carcinoma of vulva, Obstet. Gynecol. 35, 1-6 (1970).

29. Cavanagh, D., Hovadhanakal, P., and Taylor, H. B. Invasive carcinoma of the vulva: Current views on diagnosis and treatment, Mo. Med. 73, 129-135 (1976).

30. Rutledge, F., and Sinclair, M. Treatment of intraepithelial carcinoma of vulva by skin excision and graft, Amer. J. Obstet. Gynecol. 102, 806-818 (1968).

31. Merrill, J. Discussion of paper by Woodruff, J. D., Julian, C., Puray, T., Mermut, S., and Katayama, P. The contemporary challenge of carcinoma in situ of vulva. Amer. J. Obstet. Gynecol. 115, 685 (1973).

32. Collins, J. H. Discussion of paper by Parker, R. T., Duncan, I., Rampone, J., and Creasman, W. 
Operative management of early invasive epidermoid carcinoma of vulva, Amer. J. Obstet. Gynecol. 123, 354 (1975).

33. Warren, S., and Gates, O. Multiple primary malignant tumors, survey of literature and statistical study, Amer. J. Cancer 16, 1458-1514 (1932).

34. Report of the United Nations Scientific Committee on the effects of atomic radiation. Official records of General Assembly, Nineteen Sessions, Supplement No. 14 CA/5814, United Nations, New York (1964).

35. Conway, H., and Hugo, N. Radiation dermatitis and malignancy, Plas. Recons. Surg. 38, 255-268 (1966).

36. Collins, C. G., Hansen, L. H., and Thercot, E. A clinical stain for use in selecting biopsy sites in patients with vulvar disease, Amer. J. Obstet. Gynecol. 28, 158-163 (1966).

37. Broen, F. M., and Osteogard, D. R. Toluidine blue and colposcopy for screening and delineating vulvar neoplasia, Obstet. Gynecol. 38, 775-778 (1971).

38. Rubio, C. A., and Thomassen, P. A critical evaluation of Schiller test in patients before conization, Amer. J. Obstet. Gynecol. 1, 96-99 (1976). 